\title{
BMJ Open Community first response and out-of- hospital cardiac arrest: a qualitative study of the views and experiences of international experts
}

\author{
Eithne Heffernan (D) , ${ }^{1}$ Jenny Mc Sharry, ${ }^{2}$ Andrew Murphy, ${ }^{1}$ Tomás Barry, ${ }^{3}$ \\ Conor Deasy, ${ }^{4,5}$ David Menzies, ${ }^{5,6,7}$ Siobhan Masterson ${ }^{1,5}$
}

To cite: Heffernan E, Mc Sharry J, Murphy A, et al. Community first response and out-of-hospital cardiac arrest: a qualitative study of the views and experiences of international experts. BMJ Open 2021;11:e042307. doi:10.1136/ bmjopen-2020-042307

- Prepublication history and additional materials for this paper is available online. To view these files, please visit the journal online (http://dx.doi org/10.1136/bmjopen-2020042307).

Received 01 July 2020 Revised 20 January 2021 Accepted 05 March 2021
Check for updates

(C) Author(s) (or their employer(s)) 2021. Re-use permitted under CC BY-NC. No commercial re-use. See rights and permissions. Published by BMJ.

For numbered affiliations see end of article.

Correspondence to Dr Eithne Heffernan; eithneheffernan@gmail.com

\section{ABSTRACT}

Objectives This research aimed to examine the perspectives, experiences and practices of international experts in community first response: an intervention that entails the mobilisation of volunteers by the emergency medical services to respond to prehospital medical emergencies, particularly cardiac arrests, in their Iocality.

Design This was a qualitative study in which semistructured interviews were conducted via teleconferencing. The data were analysed in accordance with an established thematic analysis procedure.

Setting There were participants from 11 countries: UK, USA, Canada, Australia, New Zealand, Singapore, Ireland, Norway, Sweden, Denmark and the Netherlands.

Participants Sixteen individuals who held academic, clinical or managerial roles in the field of community first response were recruited. Maximum variation sampling targeted individuals who varied in terms of gender, occupation and country of employment. There were eight men and eight women. They included ambulance service chief executives, community first response programme managers and cardiac arrest registry managers.

Results The findings provided insights on motivating and supporting community first response volunteers, as well as the impact of this intervention. First, volunteers can be motivated by 'bottom-up factors', particularly their characteristics or past experiences, as well as 'top-down factors', including culture and legislation. Second, providing ongoing support, especially feedback and psychological services, is considered important for maintaining volunteer well-being and engagement. Third, community first response can have a beneficial impact that extends not only to patients but also to their family, their community and to the volunteers themselves.

Conclusions The findings can inform the future development of community first response programmes, especially in terms of volunteer recruitment, training and support. The results also have implications for future research by highlighting that this intervention has important outcomes, beyond response times and patient survival, which should be measured, including the benefits for families, communities and volunteers.

\section{Strengths and limitations of this study}

- This was one of the first qualitative studies to examine the perspectives and experiences of individuals who hold senior academic, clinical and managerial positions in the field of community first response.

- The qualitative design of this study facilitated the collection of rich, novel data on best practice in community first response in order to inform future research and practice in this field, including the establishment of new community first response programmes, as well as the advancement of existing programmes.

- A limitation of this study was that, while participants were recruited from a variety of regions across Europe, North America, Australia and Asia, there were no representatives from South America and Africa, who may have had different perspectives and experiences.

\section{INTRODUCTION}

Out-of-hospital cardiac arrest (OHCA) is a leading cause of mortality globally. ${ }^{1}$ In Europe and the USA, it has been estimated that just $8 \%-10 \%$ of OHCA patients survive to hospital discharge. $^{23}$ Those who survive can experience cognitive deficits and reduced quality of life. ${ }^{45}$ Improvements to the links in the Chain of Survival are associated with improvements in OHCA outcomes. ${ }^{6-8}$ The Chain of Survival is a series of actions, including early recognition of OHCA, rapid activation of the emergency medical services, early cardiopulmonary resuscitation (CPR), early defibrillation and skilled postresuscitation care. ${ }^{9} 10$ Numerous national and international initiatives have been implemented to optimise the links in this chain, such as public awareness campaigns and public access defibrillation programmes. ${ }^{611-13}$ These initiatives aim to improve OHCA outcomes by engaging community members in prehospital emergency care, including alerting the emergency 
medical services and commencing CPR and defibrillation while awaiting their arrival. ${ }^{914}$ This is especially vital in rural areas where the emergency medical services have limited capacity to substantially reduce their response times. $^{1415}$

Community first response is another important OHCA management initiative. ${ }^{16-18}$ This complex intervention entails the mobilisation of volunteers by the emergency medical services to respond to prehospital medical emergencies (eg, OHCA, stroke, choking and chest pain) in their locality. ${ }^{19}{ }^{20}$ These volunteers are known variously as community first responders (CFRs), citizen responders and lay rescuers. ${ }^{20-22}$ They can include lay people and/ or professionals, such as police officers, fire-fighters, off-duty paramedics and general practitioners. ${ }^{19} 20$ In contrast to bystanders who provide care spontaneously on witnessing an emergency, CFRs are typically affiliated with and activated by the emergency medical services. ${ }^{22} 23$ Furthermore, they tend to have completed CPR training and often have access to automated external defibrillators. ${ }^{24}$ A recent Cochrane review demonstrated that community first response programmes can increase rates of CPR or defibrillation performed prior to the arrival of the emergency medical services. ${ }^{19}$ Further research is required on additional outcomes, including survival and neurological function. ${ }^{19}$ However, there is evidence to suggest that improved response times result in improved survival. $^{2526}$

Community first response programmes have been established in many nations, including Canada, Australia, the Netherlands, Ireland and the UK. ${ }^{20} 2127-29$ However, these programmes can vary considerably between and within countries, particularly in terms of CFR organisation, dispatch, training, equipment and funding. ${ }^{24}$ Regions also differ with regard to the responsibilities given to CFRs, including using Automated External Defibrillators and responding to paediatric cases, road traffic accidents or non-injury falls. ${ }^{20} 2430$ These contrasts may be attributable to regional differences in demographics, geography, legislation, culture and resources. ${ }^{20} 2431$ Nevertheless, it may be possible to identify critical practices and features of effective Community First Response programmes that could be applied either internationally or across regions that are similar in terms of key factors (eg, geography, population). ${ }^{2024}$ Therefore, the aim of this research was to examine the perspectives, experiences and practices of international community first response experts. While previous qualitative studies explored the views of particular expert groups, including CFRs, patients and patient relatives, ${ }^{32-36}$ the present study added to the literature by consulting a group of experts who hold key clinical, managerial or academic roles in community first response. The findings could improve our understanding of this intervention and inform its future development and refinement.
METHODS

\section{Design}

This was a qualitative study, which is the optimal approach for developing an in-depth understanding of individuals' perspectives, experiences and actions. ${ }^{37} 38$ It has been reported in accordance with the Standards for Reporting Qualitative Research checklist (online supplemental file 1). ${ }^{39}$ The study was part of a multistage, mixed-methods project that aims to develop recommendations for the collection and analysis of Community First Response data. Therefore, the research paradigm was pragmatism, which entails selecting an approach that suits the research question, rather than an approach that suits a particular philosophy. ${ }^{40}$ The specific qualitative approach was phenomenology, or the close examination of individual experiences and perceptions of a phenomenon of interest. $^{41}$

The qualitative method was the semistructured interview. This is a conversation between a researcher and one or more participants that is based on a flexible interview schedule (online supplemental file 2). ${ }^{37} 42$ This flexibility enables the researcher to build rapport, explore unanticipated responses, discuss complex subjects and identify issues that are important to the interviewees. ${ }^{37} 43$ The interviews were primarily individual (ie, one-to-one) interviews, as this is the optimal approach for the collection of detailed accounts and the development of rapport and trust, which helps participants to speak freely and to discuss sensitive issues. ${ }^{37} 4244$ Paired interviews were utilised in cases where a participant recommended that a colleague join the interview on the basis that they had different areas of expertise or roles within their organisation and thus that they could provide more comprehensive information and insights as a pair. The use of individual and paired interviews can be considered a form of triangulation. Triangulation refers to employing multiple techniques and/or obtaining multiple perspectives to enhance the trustworthiness or validity of a qualitative study. ${ }^{445}$

\section{Participants}

The participants were a group of community first response subject matter experts (SMEs) who were employed in academic, clinical and managerial roles in this field. The inclusion criteria were self-reported ability to give informed consent, good standard of written and spoken English, minimum age of 18 years, and occupation in the community first response field. Participants were recruited from the professional network of the research team via email. Maximum variation sampling, a form of purposeful sampling, was used. ${ }^{46} 47$ This involved recruiting participants who varied in terms of the key characteristics of gender, occupation and country/region of employment. In particular, participants who held senior positions in community first response programmes, ambulance services, cardiac arrest registries and universities were sought. Sampling ceased once maximum variation and saturation had been achieved. Saturation is the 
Table 1 Demographic information of the subject matter experts SME(s)

\begin{tabular}{lll}
\hline ID code & Country of employment & $\begin{array}{l}\text { Occupational } \\
\text { category }\end{array}$ \\
\hline SME1 & Netherlands & Researcher \\
SME2 & Australia & Manager \\
SME3 & Singapore & Researcher/manager \\
SME4 & Sweden & Researcher/clinician \\
SME5 & Canada & Researcher/clinician \\
\hline SME6 & Norway & Researcher/clinician \\
SME7 & New Zealand & Manager/clinician \\
\hline SME8 & UK (Northern Ireland) & Manager \\
SME9 & Ireland & Manager/clinician \\
SME10 & USA & Researcher \\
SME11 & USA & Researcher/clinician \\
SME12 & Netherlands & Manager/clinician \\
\hline SME13 & New Zealand & Researcher \\
SME14 & UK (England) & Manager \\
SME15 & Denmark & Manager/clinician \\
\hline SME16 & UK (England) & Manager \\
\hline & & \\
\hline
\end{tabular}

point at which no new patterns or salient information are uncovered from the data. ${ }^{47}$ Saturation was assessed through preliminary data analysis and a discussion among the research team.

Of the 27 SMEs who were contacted about the study, 16 consented to participate. Each participant was assigned a unique identification code (see table 1). There were eight men and eight women. They included managers and engagement officers for community first response programmes, ambulance service chief executives, cardiac arrest registry coordinators and research department directors. Five individuals provided a reason for declining to participate in the study. Three recommended a colleague with more relevant expertise in their stead, while two were unavailable due to work commitments.

\section{Procedure}

Potential participants were sent a study invitation email and a participant information sheet, which provided them with detailed information about the study. They were given the opportunity to contact the research team with any questions about the study. Informed, written consent was obtained from each participant. Subsequently, they participated in an interview via teleconferencing at a time and location (eg, home, office) of their choosing. Each interview lasted approximately 1 hour. Ten interviews were video calls, while six were audio-only calls. Twelve participants were interviewed individually. Four participants opted to be interviewed in pairs with a colleague. The interviews were conducted by the first author: a postdoctoral researcher in the Discipline of General Practice, School of Medicine, National University of Ireland
(NUI), Galway. She had formal training in and prior experience of conducting qualitative studies, including interviewing academics and clinicians. ${ }^{48-50}$ The interviews were audio-recorded and transcribed verbatim. The data collected were treated confidentially and stored securely (eg, locked cabinets, password-protected computers) in NUI Galway.

\section{Patient and public involvement}

The multistage, mixed-methods project, of which this study is part, has a panel of three patient and public involvement representatives who advise on research design and dissemination. Furthermore, the interview schedule and procedure of this study were refined based on feedback from three SMEs from the professional network of the research team.

\section{Data analysis}

The first author conducted the analysis in accordance with Braun and Clarke's thematic analysis procedure, which aims to develop a description of the patterns of response in the dataset that capture important information about the research question. ${ }^{51}$ The procedure entails becoming immersed in the data, generating initial codes, searching for themes, reviewing themes, defining and naming themes, and producing the written report. QSR International NVivo V.12 software supported this process. The analysis was inductive, such that the codes and themes were based on the data collected, rather than on an existing framework. ${ }^{5152}$ This approach was preferred to deductive analysis, which can overlook key data that do not fit with the selected framework. ${ }^{5152}$

Peer debriefing was used to enhance the trustworthiness of the analysis. ${ }^{45354}$ Specifically, the second author independently analysed five transcripts and then had several meetings with the first author to compare their findings. Some minor discrepancies were resolved through discussion and a review of the data. No substantial differences between their interpretations were identified, suggesting that the analysis was not limited to the perspective of a single researcher. To further bolster trustworthiness, disconfirming evidence analysis was performed. ${ }^{45} 5354$ Once preliminary themes were identified, the first author searched for any data that contradicted them. She then ensured that the final themes had sufficient supportive evidence and included any pertinent disconfirming evidence in the written report. ${ }^{45} 5354$

\section{RESULTS}

The results showed that there are diverse community first response models across the different countries. For example, in some regions, lay CFRs are organised in teams of volunteers from the same community, while in other regions, lay CFRs act independently of one another, rather than as part of a group. In addition, there is considerable regional variation in the type and amount of support offered to CFRs. Though some regions have 


\begin{tabular}{|c|c|c|}
\hline Theme & Subtheme & Summary \\
\hline \multirow{2}{*}{$\begin{array}{l}\text { Motivation of } \\
\text { community first } \\
\text { responders }\end{array}$} & $\begin{array}{l}\text { Bottom-up } \\
\text { motivation }\end{array}$ & Responder motivation can be influenced by their personality traits and past experiences. \\
\hline & $\begin{array}{l}\text { Top-down } \\
\text { motivation }\end{array}$ & Responder motivation can be influenced by the culture and society surrounding them. \\
\hline \multirow{2}{*}{$\begin{array}{l}\text { Support for } \\
\text { community first } \\
\text { responders }\end{array}$} & Feedback & $\begin{array}{l}\text { Peer and/or professional feedback can improve responders' knowledge and skills and } \\
\text { provide valuable reassurance and recognition. }\end{array}$ \\
\hline & $\begin{array}{l}\text { Psychological } \\
\text { support }\end{array}$ & $\begin{array}{l}\text { Peer and/or professional psychological support is required by some responders who } \\
\text { have experienced distress and can enable them to continue in the role. }\end{array}$ \\
\hline \multirow{4}{*}{$\begin{array}{l}\text { Impact of } \\
\text { community first } \\
\text { response }\end{array}$} & $\begin{array}{l}\text { Impact on } \\
\text { patients }\end{array}$ & $\begin{array}{l}\text { Community first response is thought to improve patient outcomes, though additional } \\
\text { research is needed to fully understand its impact on patients. }\end{array}$ \\
\hline & $\begin{array}{l}\text { Impact on } \\
\text { relatives }\end{array}$ & $\begin{array}{l}\text { Community first response can be an important source of support and comfort for } \\
\text { patients' relatives. }\end{array}$ \\
\hline & $\begin{array}{l}\text { Impact on } \\
\text { communities }\end{array}$ & $\begin{array}{l}\text { Community first response can be a valuable resource and can strengthen cohesion and } \\
\text { resilience within a community. }\end{array}$ \\
\hline & $\begin{array}{l}\text { Impact on } \\
\text { community first } \\
\text { responders }\end{array}$ & $\begin{array}{l}\text { Community first response can be rewarding for the responders themselves, such as by } \\
\text { giving them a sense of pride and membership of a team. }\end{array}$ \\
\hline
\end{tabular}

comprehensive support services, such as debriefing and counselling, other regions are still in the process of developing them. The results also showed that community first response programmes vary across the different countries in terms of complexity, with some having one main type of CFR and others comprising multiple categories of CFR. For instance, there are several types of CFR in the Ireland, including laypersons, general practitioners, police officers and fire-fighters. Furthermore, while in some regions CFRs are highly integrated with the emergency medical services, in other regions they are considered a relatively separate group. Despite the diversity of the community first response systems, there were key patterns within the participants' responses, which resulted in the identification of three primary themes and a variety of subthemes (see table 2).

\section{Theme 1: motivation of CFRs}

It was reported that the motivation to volunteer as a CFR can come from within the individual, particularly their characteristics or past experiences (ie, bottom-up motivation), as well as from the society and culture surrounding that individual (ie, top-down motivation).

\section{Bottom-up motivation}

The participants put forth an array of factors that prompt individuals to join community first response programmes. First, many volunteer because they are altruistic and empathetic in nature: 'These people are actually so motivated by helping other people... Even if it's just coming just after the ambulance arrival and then supporting the family... They feel that they can [make] a difference,' (SME15). In addition, some CFRs are inspired by their personal experiences or family history: 'Some of them have got a real drive to become a CFR because... somebody that they're close to has undergone... a cardiac arrest and they've seen the benefit of them being helped, (SME14). There are also those who volunteer because they need a social outlet: 'Some... do it as part of a social experience... It's another social avenue,' (SME2). Others hope that volunteering will help them to achieve their career goals: 'There's a strand of people who feel that maybe it will help with their career progression or they want to have on their CV that they're volunteering and... they're up to date with their training,' (SME8).

Several participants said that CFRs commonly 'Want to support their local communities... That's one big motivator... It's about giving back to their local communities,' (SME16). Some reported that CFRs are often pillars of the community. SME9 said: 'Isn't it always the same people in... your local village [who get involved] in the church and the school and everything?... It's that sort of person.' SME9 added that such individuals are crucial to establishing and maintaining CFR schemes in their communities: 'It takes one main person... your doctor, your priest, your school teachers... Whoever the leader is... within an area that people look up to... You need those kinds of people... to champion it.' Furthermore, some feel that they have a responsibility to volunteer due to their qualifications or status, such as healthcare professionals, lifeguards, or police officers: 'Some people feel obligated out of a sense of duty... They have been trained, they hold a position within the community,' (SME2).

A small number of participants noted that a minority of people want to become a CFR because they seek excitement or attention. Such individuals may not be permitted to join a Community First Response programme, especially if they do not adjust their expectations following initial training. According to SME8: 'There's a theme of people who want... the excitement and the adrenaline rush... They think they're going to have blue lights and... 
be a paramedic... It tends to attract, in the minority, that kind of person'. Another participant, SME6, said that a minority volunteer 'Because they want to go to the media afterwards... or... they feel very inadequate or they want to show-off... Some... enjoy the attention... They try to do more than what they have been trained to. There are not too many, but some will always show up.'

\section{Top-down motivation}

Societal and cultural factors can influence one's motivation to volunteer as a CFR. SME10 explained: 'A lot of our participating communities... have this culture of making cardiac arrest... a priority... They've really found a way to engage their population... in bystander CPR... There's... two ways to go about it: individual-driven and... this more top-down approach.' Various organisations, such as government bodies, charities and academic institutions, can encourage involvement in Community First Response. SME9 said: 'Some [CFR] groups are... supported by voluntary agencies.' SME3, from Singapore, stated: 'There is a national... save-a-life initiative... The government... are teaching CPR, they are teaching first aid... There's this real sense of being part of the welfare and security of your country.' Furthermore, some regions have made it compulsory to engage in aspects of community first response. SME15 provided an example from Denmark: 'It is mandatory in schools to teach CPR. It is mandatory to have a CPR course when you take a driver's licence.' Additionally, SME3 said: 'Because Singapore has obligatory military service for males, every male of a certain age has undergone CPR... training.'

Several participants proposed that rural communities have a culture that fosters participation in Community First Response. SME6 gave an example from Norway: 'There are big areas... where the ambulance uses quite a long time to get there. We have had a tradition for helping each other out for a long time... Neighbours would help neighbours... Communities... would come together on different days and help each other.' SME12, from the Netherlands, commented: 'Out in a rural part... already people were... very attuned to this job.' His region capitalised on this when establishing a community first response programme:

We started... in the most rural communities where people know that... they are depending on themselves... Already quite a lot of people... were trained to do the CPR... but... we didn't have the system to get the message to them that they were needed. So... one community after another, we connected them to the system.

Societal and cultural factors can also deter people from engaging in community first response. In particular, in some areas, first response is regarded as the domain of healthcare professionals, rather than volunteers:

The greatest barriers to implementing community response are... legislation barriers. For example, in
Ontario... the Ambulance Act... does not authorise the dispatch of any... volunteer or non-professional provider... Changing culture too, as... people in the communities... expect a professional responder. Changing the culture of the paramedics in that they... want to keep ownership of this, (SME5).

Volunteers may be viewed as a risk to patient safety and privacy. SME11 said: 'In the US... they only will notify someone... if it's a public event... for safety reasons. [In] other countries... there's less security concerns, there's a different culture, and they respond to... all events... in a public location or residential.' There were similar issues in Canada:

The decision makers... are used to thinking about 'worst case scenario'... so there are... concerns around... volunteers using the [CFR alert] app to steal from people who have been taken away to the hospital, the media using the app to come to the scene and get a good story... There's been visions of too many people on the scene... and the paramedics can't get to the patient, (SME5).

It is possible to shift this culture over time, according to SME12: 'Now everybody is convinced but, at the time, they were really thinking 'It's a mad idea... We're the professionals and we don't need the lay people to do this job.'... Many years further... in the whole of the Netherlands, we have this system [of] lay rescuers.'

\section{Theme 2: support for CFRs}

The provision of ongoing support, especially feedback and psychological services, was regarded as key to maintaining the well-being and engagement of CFRs.

\section{Feedback}

The majority of participants reported that it is important to provide feedback to CFRs, as it improves their knowledge and skills and also helps them to feel reassured and appreciated. 'The one thing that CFRs want is really good, effective communication and to be kept in the loop with... the CFR world and the ambulance service world... It makes them feel valued,' (SME16). CFRs particularly welcome guidance from emergency medical services personnel. For example, SME9 found that CFRs in her region appreciated having contact with an Engagement Officer from the statutory ambulance service: 'They [said] 'It's amazing to know that there's somebody there.'... They felt... more supported and cared for.' CFRs can also benefit from peer support. SME15 provided an example:

We have established... a Facebook page for the first responders... The idea was to have [them] ask questions and we would then... give answers... It turned out that [they] provided all the right answers... so it's seldom now that we actually intervene... They are very supportive in telling people: 'You did the right thing,' and 'Nothing else you could do,' and 'Great what you have achieved.' 
It is important that CFRs feel encouraged, rather than disheartened, by any feedback received. For instance, SME1 said that care must be taken when providing feedback on CPR quality to lay CFRs: 'You can't blame [them] for doing something not in a perfect way, because it's... already good that they performed CPR, so you need to be a little bit conscious.' SME15 said: 'We don't... assess the quality of the CPR... It's just attending and trying to save a life that matters. It's a trust basis.'

CFRs appreciate learning about the overall progress and outcomes of their Community First Response programme: 'Share the data, whether it's... just the highlevel view of how things are trending, because people like to see that whatever they're part of is doing well,' (SME3). This can include sharing data via reports, newsletters, meetings, or conferences. For example, SME3 said: 'We have... the 'Survivor Awards'... to bring... people who were resuscitated... together with... everybody prehospital that played a role in saving their lives and it's a wonderful thing to see. The [CFRs] really feel fulfilled.' Furthermore, many CFRs are keen to learn the outcomes of specific cases in which they were involved. However, this often raises concerns about patient privacy and wellbeing. SME12 said: 'Often they want to have contact with the patient to see how he's doing. Some patients don't want to have contact with the [CFR]'. SME2 said: 'In terms of providing feedback on the outcome of the patient... we have very strict ethics approval... We can't report it at anything other than a population level. We're... getting that changed so that anyone... involved in the care of a patient can follow the outcome of that patient.' SME4 was of the view that CFRs should learn of patient outcomes: 'They have the right to know what happened... If you don't get feedback, you never know what's right or wrong.'

\section{Psychological support}

The participants agreed that responding to emergencies can affect the mental well-being of CFRs. It was reported that, though many CFRs do not experience notable distress or trauma, support should be provided to those who require it: 'They need... to be able to talk to somebody. Not everybody needs it, but the opportunity needs to be there... Most patients with cardiac arrest actually die and this is quite traumatic,' (SME6). Psychological support could be especially important for lay CFRs: 'We're talking about your average citizen... They're not medical people. They may not see this end-of-life... or emergency situation every day. It could be quite traumatic,' (SME3). Furthermore, some emergencies could be more distressing than others: 'Particularly if it's a pretty difficult call: children... or... in smaller communities... when they're responding to somebody they know,' (SME11). Whether or not an emergency causes distress ultimately depends on the individual CFR: 'What might stress one individual might not really affect another,' (SME14). Therefore, psychological support should be widely accessible: 'There should be ongoing availability. It shouldn't just be an extraordinary event,' (SME11).
An appropriate psychological support system can allow CFRs who have experienced distress to continue participating in the programme: 'It's about having the right support structure around them to allow that healing to occur... and... people having trust in the system that it's not going to be used against them... It's completely separate. It's totally confidential,' (SME2). Several participants regarded debriefing as an important component of psychological support: 'Deconstructing the event, going through it, and allowing people to just participate-that often helps,' (SME11). Professionals, such as emergency medical services personnel, can facilitate debriefing. SME7 said: 'They will... talk through the case... to give them feedback at the time to allay concerns... That actually solves most concerns and anxieties... if that's done well at the time.' Peers can also facilitate debriefing, particularly in regions where CFRs are organised in teams: 'The team can support each other... in the event of a more difficult job... The team-based system has real advantages,' (SME14). Several participants highlighted the importance of formal mental health services (eg, counselling, Critical Incident Stress Management), especially in cases where initial debriefing proves insufficient. SME2 described services for lay CFRs in his region: 'We... link them in with better care if they need it... via phone call close to 24 hours after the event... We can refer the person to their local doctor,... five no-cost counselling sessions, [or] the crisis counsellor can go out and discuss with the person.' Some regions are still in the process of developing such systems. For example, SME6 said: 'It's really, very unstructured... It wasn't... really quality controlled, but that's getting better.'

\section{Theme 3: impact of CFRs}

It was reported that community first response has a beneficial impact that can extend not only to patients but also to their family, their community and to the CFRs themselves.

\section{Impact on patients}

The participants reported that community first response can improve patient outcomes. For example, SME15 explained that it has played an important role in improving response times and survival rates for OHCA patients in his region:

We have been doing a lot of research on how to improve cardiac arrest survival. We have tripled survival within $10-15$ years... Part of that success have been the initiatives... on engaging the community in first responders and dissemination of [defibrillators] and awareness in the public... Actually $40 \%$ of cardiac arrests: the volunteer first responder gets there before the ambulance.

SME9 stated that Community First Response is an important link in the Chain of Survival, especially in terms of increasing Return of Spontaneous Circulation (ROSC) rates: 
There's an awful lot of the out-of-hospital cardiac arrests... where we're getting ROSCs and there's been mention [of] the Community First Responder... attending... before the ambulance... We can see... everyone having their own part to play, but... we need to look at the data to see can we prove that.

Though research has been conducted on community first response in many regions, several participants suggested that additional investigations are required to better understand its impact on patient outcomes. For example, SME15 said: 'It does make a difference and... we increase survival. There's no doubt about that. But what is the most efficient intervention...? Is it having a layperson who has a CPR certificate... or is it telephone-assisted CPR or is it the professional first responders?' Some participants said that more work is needed to pinpoint the specific contribution of community first response to OHCA survival, as it is just one of several links in the chain. SME16 said: 'Until recently, we hadn't collected a huge amount of data on our CFRs... We... have the overall... survival figures... but I couldn't pick out of that what's down to a CFR, at the moment... We've got to... work out how we're going to achieve that.' It is also important to investigate outcomes other than survival:

Survival is, of course, very important, but I don't think it's that important to the patients. It's more if you're able to do your daily chores, live at home, and... have a normal life... I'd really like to know about shortterm memory loss and how this affects the patients and the... family, (SME6).

\section{Impact on relatives}

Many participants reported that community first response can be beneficial for patients' families. In particular, CFRs often provide valuable support and comfort to relatives during emergencies: 'Sometimes it's about what they can do for a family... which can't be measured really... Sometimes it's actually about the reassurance to family members, to calm them down, to get the information of what happened,' (SME9). Relatives tend to appreciate receiving assistance and seeing that every effort is being made to help the patient: 'When people come to help, the family is usually very positive. They like to see that people are doing a good job and trying to help,' (SME6). CFRs make an important contribution by supporting the family, even in cases where they arrive after the ambulance crew or where the patient does not survive:

If we get an ambulance crew to the scene prior to the CFR... they [still] have a really valuable role in being able to manage and support the family in... one of their greatest times in need... Whether the patient is taken to hospital or whether they're perhaps declared deceased at the scene,... they can play a pivotal role in... support functions immediately after the event, (SME16).

\section{Impact on communities}

Several participants proposed that community first response programmes can be advantageous for communities: 'The feedback... from communities is very positive... People are very grateful for... support in times of need,' (SME16). In particular, these programmes can provide communities with valuable knowledge and skills, in addition to strengthening their cohesion and resilience:

It might be difficult to measure but... it might help increase feelings of community cohesiveness, feelings of... neighbours helping neighbours, a feeling of safety and security... If the programme is successful in reducing death and disability,... there will be economic benefits for the family and the community... besides, of course, the emotional... benefits... It can raise awareness of cardiac arrest,... especially if the [CFRs] behave like ambassadors in the community... Many of them set up their own CPR training events... so there's... spin-off benefits, (SME5).

Furthermore, community first response programmes can be developed for the benefit of communities who have specific needs, as shown by an example from SME2: 'The Jewish population have a Jewish first response service here in Melbourne called Hatzolah and there's a degree of cultural ease having them in attendance in addition to the paramedics. So having [responders] who are familiar to them can provide a bit of comfort.'

Some participants from regions where CFRs are organised in teams within their local communities proposed that there are both advantages and disadvantages to this approach. SME8 explained that it could be difficult for CFRs and patients' relatives to encounter one another regularly: 'There's very much a community spirit to it... Everybody's helping everybody... The downside... is that they still have to live in the area where that person... passed on and they have to meet those people and those people have to meet them.' Additionally, SME9 said:

The last thing that you want is... someone in their time of need and [a CFR] that they've been fighting with for the last ten years would [come] to their door... There's a responsibility on the group to get out there and let people know... 'When the ambulance service is called... it... could be us that could come.

It is also important for CFRs to ensure that community members have realistic expectations: 'The people in society... think often that first responders can do more than they can.' (SME4).

\section{Impact on CFRs}

Several participants noted that community first response can be rewarding for the volunteers themselves. In particular, they can obtain a sense of pride and fulfilment: 'They're so proud of actually being part of that system [that] helped somebody.' (SME15). Additionally, they 
can feel better prepared for emergencies in their own home:

People get some... peace of mind, having completed a training, knowing that... the person that you use your training on could very well be your... loved one, so there's a benefit there... We... as part of our training... make sure folks see the importance of what they're doing [and] that they're part of something... special. (SME3).

Finally, in regions where CFRs are organised in groups, they often enjoy being part of a team: 'You're one of the team who is doing this great job... People are very happy to be in this network.' (SME12).

\section{DISCUSSION}

This study examined the perspectives and experiences of international community first response experts to identify features or practices that could be used to develop and refine this intervention. The results provided insights on CFR motives that have implications for their recruitment and training. It was found that CFR motivation is influenced by their personal characteristics and past experiences, such as having an altruistic personality or previously witnessing an emergency. This finding is supported by past research on the experiences of CFRs. ${ }^{34-365556}$ The current study suggests that a minority of CFRs seek excitement or attention. This aligns with a previous qualitative study of lay CFRs, which reported that some are attracted to the dramatic aspects of the role. ${ }^{35}$ Consequently, community first response programmes tend to carefully select and train their volunteers. A novel finding of the present study is that societal and cultural factors can influence CFR motivation, such as public awareness campaigns, rural traditions and legislation.

This study highlighted the importance of providing ongoing support for maintaining CFR well-being and engagement. In particular, the results show that feedback from professionals and/or peers can be a crucial source of guidance and reassurance for CFRs. Previous qualitative research found that CFRs desire more feedback, including acknowledgement of their efforts, reassurance regarding their performance during emergencies and information on patient outcomes. ${ }^{20} 5758$ However, feedback must be provided with care so that patient privacy is protected and so that CFRs are not discouraged, especially when patients do not survive. ${ }^{57}{ }^{58}$ Furthermore, a past study on ambulance volunteers and first responders in Australia and New Zealand suggests that they should not only receive but also provide feedback, including having input into the decision-making processes of ambulance services. ${ }^{59}$ In addition to feedback, the current study found that psychological services are needed to support CFRs who experience distress or even trauma. Previous studies confirm that some CFRs have adverse psychological experiences, including sleep disturbance, intrusive thoughts and weight loss. ${ }^{345760-62}$ The present study suggests that debriefing shortly following an emergency may be beneficial for CFRs. Those who continue to experience distress should be referred to formal mental health services. There is some evidence in the literature to support this approach. ${ }^{58} 63$ This study, coupled with a survey of European OHCA experts, demonstrated that regions vary greatly in terms of the type and amount of support offered to CFRs. ${ }^{24}$ In some regions, little support is available, particularly for lay CFRs. Additional investigations are required to identify the most effective means of supporting CFRs, such as research on the benefits of peer-led debriefing.

Finally, this study showed that community first response can benefit not only patients but also their families, their communities and the CFRs themselves. In particular, it can be an important source of care and comfort for patients and their relatives, it can provide knowledge, skills, resilience and cohesion to communities, and it can give CFRs a sense of pride, fulfilment and social connection. Previous research on volunteering indicates that it can be advantageous to both the individual volunteers and their communities by generating and enhancing social capital, which refers to the social networks, connectedness, trust, empowerment and resources that can result from individuals within a community coordinating and cooperating to achieve a common goal. ${ }^{64}$ There is evidence to suggest that social capital can, in turn, improve health and quality of life at both the individual level and the community level. ${ }^{656}$ Previous research specifically on community first response found that CFRs cited contributing to their community as a key motivation and that they consider supporting patients' families to be a significant aspect of the role. ${ }^{3536}$ Furthermore, emergency medical services personnel have reported that it is often challenging to balance caring for both patients and patients' families. ${ }^{67}$ Therefore, the care provided by CFRs is valuable, even when the emergency medical services are first to arrive on scene or when the patient does not survive. To date, evaluations of community first response have focused on the outcomes of responses times and survival. ${ }^{19}$ The impact on families, communities, and CFRs tends to go unmeasured. The present study suggests that these more holistic outcomes should be considered when seeking to comprehensively assess the value of community first response, though they could prove somewhat difficult to measure. Therefore, future research should identify all of the key outcomes of this intervention, as well as the most appropriate means of measuring them.

A limitation of this study was that, though there were participants from a variety of regions in Europe, North America, Australia and Asia, there were no participants from South America and Africa. Furthermore, the 11 countries represented in this study were all ranked among the top 15 nations on the Human Development Index. ${ }^{68}$ Participants from countries 
with lower Human Development Index rankings may have provided different perspectives. It should also be noted that, as this was a qualitative study in which sampling ceased once maximum variation and saturation had been achieved, some countries with established community first response programmes (eg, Finland, Italy) were not represented. Additionally, CFRs themselves were not consulted as part of this study. However, previous qualitative studies in this field have been conducted with CFRs, as well as with patients and patients' relatives. ${ }^{32-34}$ The present study focused on community first response academics, clinicians and managers from a range of countries because few, if any, past qualitative studies have been carried out with this population.

Another potential limitation of this study is that the paired interview participants could have found it more difficult to express themselves openly compared with the individual interview participants due to being in the presence of a colleague. For example, they could have felt somewhat obliged to present a favourable view of their organisation. In addition, the participants, who included community first response programme managers, reported largely positive views of this intervention, such as its beneficial impact on multiple stakeholders (eg, patients, communities and CFRs). It is possible that a different sample would provide an alternative perspective on community first response. For example, two past studies found that first responders felt that their role was sometimes undervalued by or unclear to emergency services personnel and/or members of the public. ${ }^{3469}$ Another study found that salaried staff can be resistant to the integration of volunteers within ambulance services. ${ }^{59}$ Therefore, future research should consult different stakeholders who could have varied attitudes towards community first response (eg, paramedics, nurses and the public). Another avenue for future research would be to develop a theoretical framework of best practice in community first response. Though the present study provided a rich description of the perspectives and experiences of experts in community first response, the development of a best practice model or theory would be a valuable addition to the literature. Nevertheless, the current study was a novel investigation of the experiences and opinions of international community first response researchers and practitioners that produced new insights on motivating and supporting CFRs, as well as the benefits of community first response for a variety of stakeholders. These findings can be used to guide future research and practice in this field, including the establishment of new community first response programmes and the advancement of existing programmes.

\section{Author affiliations}

${ }^{1}$ Discipline of General Practice, Clinical Science Institute, School of Medicine, National University of Ireland Galway, Galway, Ireland
${ }^{2}$ Health Behaviour Change Research Group, School of Psychology, National University of Ireland Galway, Galway, Ireland

${ }^{3}$ School of Medicine, University College Dublin, Dublin, Ireland

${ }^{4}$ Department of Emergency Medicine, Cork University Hospital, Cork, Ireland

${ }^{5}$ National Ambulance Service, Health Service Executive, Dublin, Ireland

${ }^{6}$ St Vincent's University Hospital, Dublin, Ireland

${ }^{7}$ CFR Ireland, Dublin, Ireland

Twitter Jenny Mc Sharry @jenmcsharry and David Menzies @David_menzies

Acknowledgements The research team would like to thank the participants and Public and Patient Involvement Representatives who contributed to this research. We would also like to thank our collaborators for their assistance with the planning and conduct of this research, including Mark Callanan and Professor Cathal

O'Donnell of the National Ambulance Service, Ireland, as well as Jacqueline Egan of the Pre-Hospital Emergency Care Council, Ireland.

Contributors All of the authors meet the ICMJE criteria for authorship. SM and EH designed the study with input from the other authors (JMS, AM, TB, CD and DM). EH and SM recruited the participants. EH acquired the data. EH analysed the data with assistance from JMS. EH drafted the manuscript. All authors contributed to the interpretation of the data (EH, JMS, AM, TB, CD, DM and SM). All authors critically revised the manuscript for important intellectual content, approved the final version, and agreed to be accountable for all aspects of the work (EH, JMS, AM, TB, CD, DM and SM).

Funding This work was supported by an Applied Partnership Award from the Health Research Board, Ireland in partnership with the National Ambulance Service, Ireland and the Pre-Hospital Emergency Care Council, Ireland (Grant number: APA-2016-1859).

Competing interests TB is an academic general practitioner with roles in cardiac arrest education, research and clinical care. He declares no other competing interests. The remaining authors have no competing interests to declare.

Patient consent for publication Not required.

Ethics approval The study was approved by the Research Ethics Committee of the National University of Ireland (NUI), Galway.

Provenance and peer review Not commissioned; externally peer reviewed.

Data availability statement № additional data are available.

Supplemental material This content has been supplied by the author(s). It has not been vetted by BMJ Publishing Group Limited (BMJ) and may not have been peer-reviewed. Any opinions or recommendations discussed are solely those of the author(s) and are not endorsed by BMJ. BMJ disclaims all liability and responsibility arising from any reliance placed on the content. Where the content includes any translated material, BMJ does not warrant the accuracy and reliability of the translations (including but not limited to local regulations, clinical guidelines, terminology, drug names and drug dosages), and is not responsible for any error and/or omissions arising from translation and adaptation or otherwise.

Open access This is an open access article distributed in accordance with the Creative Commons Attribution Non Commercial (CC BY-NC 4.0) license, which permits others to distribute, remix, adapt, build upon this work non-commercially, and license their derivative works on different terms, provided the original work is properly cited, appropriate credit is given, any changes made indicated, and the use is non-commercial. See: http://creativecommons.org/licenses/by-nc/4.0/.

ORCID iD

Eithne Heffernan http://orcid.org/0000-0003-4485-7658

\section{REFERENCES}

1 Myat A, Song K-J, Rea T. Out-of-hospital cardiac arrest: current concepts. Lancet 2018;391:970-9.

2 Benjamin EJ, Virani SS, Callaway CW, et al. Heart disease and stroke statistics -2018 update: a report from the American heart association. Circulation 2018;137:e67-492.

3 Gräsner J-T, Wnent J, Herlitz J, et al. Survival after out-of-hospital cardiac arrest in Europe - Results of the EuReCa TWO study. Resuscit 2020;148:218-26.

4 Lim C, Verfaellie M, Schnyer D, et al. Recovery, long-term cognitive outcome and quality of life following out-of-hospital cardiac arrest. J Rehabil Med 2014;46:691-7. 
5 Green CR, Botha JA, Tiruvoipati R. Cognitive function, quality of life and mental health in survivors of our-of-hospital cardiac arrest: a review. Anaesth Intensive Care 2015;43:568-76.

6 Nolan JP, Perkins GD, Soar J. Improving survival after out-of-hospital cardiac arrest. BMJ 2015;351:h4989.

7 Strömsöe A, Svensson L, Axelsson Åsa B, et al. Improved outcome in Sweden after out-of-hospital cardiac arrest and possible association with improvements in every link in the chain of survival. Eur Heart J 2015;36:863-71.

8 Hasselqvist-Ax I, Riva G, Herlitz J, et al. Early cardiopulmonary resuscitation in out-of-hospital cardiac arrest. $N$ Engl $J$ Med 2015:372:2307-15.

9 Nolan JP, Hazinski MF, Aickin R, et al. Part 1: Executive summary: 2015 international consensus on cardiopulmonary resuscitation and emergency cardiovascular care science with treatment recommendations. Resuscitation 2015;95:e1-31.

10 Deakin CD. The chain of survival: not all links are equal. Resuscitation 2018;126:80-2.

11 Wissenberg M, Lippert FK, Folke F, et al. Association of national initiatives to improve cardiac arrest management with rates of bystander intervention and patient survival after out-of-hospital cardiac arrest. JAMA 2013;310:1377-84.

12 Perkins GD, Lockey AS, de Belder MA, et al. National initiatives to improve outcomes from out-of-hospital cardiac arrest in England. Emerg Med J 2016;33:448-51.

13 Winkle RA. The effectiveness and cost effectiveness of public-access defibrillation. Clin Cardiol 2010;33:396-9.

14 Monsieurs KG, Nolan JP, Bossaert LL, et al. European resuscitation council guidelines for resuscitation 2015: section 1. executive summary. Resuscitation 2015;95:1-80.

15 Ong MEH, Perkins GD, Cariou A. Out-of-hospital cardiac arrest: prehospital management. Lancet 2018;391:980-8.

16 Masterson S, Wright P, O'Donnell C, et al. Urban and rural differences in out-of-hospital cardiac arrest in Ireland. Resuscitation 2015;91:42-7.

17 Hollenberg J, Svensson L, Rosenqvist M. Out-of-hospital cardiac arrest: 10 years of progress in research and treatment. $J$ Intern Med 2013;273:572-83.

18 Truong HT, Low LS, Kern KB. Current approaches to cardiopulmonary resuscitation. Curr Probl Cardiol 2015;40:275-313.

19 Barry T, Doheny MC, Masterson S, et al. Community first responders for out-of-hospital cardiac arrest in adults and children. Cochrane Database Syst Rev 2019;7:CD012764.

20 Phung V-H, Trueman I, Togher F, et al. Community first responders and responder schemes in the United Kingdom: systematic scoping review. Scand J Trauma Resusc Emerg Med 2017;25:58

21 Zijlstra JA, Stieglis R, Riedijk F, et al. Local lay rescuers with AEDs, alerted by text messages, contribute to early defibrillation in a Dutch out-of-hospital cardiac arrest dispatch system. Resuscitation 2014;85:1444-9.

22 Heffernan E, Oving I, Barry T, et al. Factors that motivate individuals to volunteer to be dispatched as first responders in the event of a medical emergency: a systematic review protocol. HRB Open Res 2019;2:34.

23 Whittaker J, McLennan B, Handmer J. A review of informal volunteerism in emergencies and disasters: definition, opportunities and challenges. Int J Disaster Risk Reduct 2015;13:358-68.

24 Oving I, Masterson S, Tjelmeland I, et al. Inventory of firstresponse treatments after out-of-hospital cardiac arrest in Europe. Resuscitation 2019;142:e2-3.

25 O'Keeffe C, Nicholl J, Turner J, et al. Role of ambulance response times in the survival of patients with out-of-hospital cardiac arrest. Emerg Med J 2011;28:703-6.

26 Bürger A, Wnent J, Bohn A. The effect of ambulance response time on survival following out-of-hospital cardiac arrest: an analysis from the German resuscitation registry. Dtsch Arztebl Int 2018:115:541.

27 O'Meara P. The prehospital community-volunteer model has a place in rural Australia. Australas J Paramed 2014:1.

28 Orkin AM, VanderBurgh D, Ritchie SD, et al. Community-based emergency care: a model for prehospital care in remote Canadian communities. CJEM 2016;18:385-8.

29 Masterson S, Robinson E, Wright P, et al. Community cardiac first responders in Ireland. Resuscitation 2013;84:S33.

30 Campbell A, Ellington M. Reducing time to first on scene: an ambulance-community first responder scheme. Emerg Med Int 2016;2016:1-7.

31 Orkin A, Vanderburgh D, Born K, et al. Where there is no paramedic: the Sachigo lake wilderness emergency response education initiative. PLoS Med 2012;9:e1001322.
32 Bremer A, Dahlberg K, Sandman L. To survive out-of-hospital cardiac arrest: a search for meaning and coherence. Qual Health Res 2009;19:323-38.

33 Ann-Britt T, Ella D, Johan $\mathrm{H}$, et al. Spouses' experiences of a cardiac arrest at home: an interview study. Eur J Cardiovasc Nurs 2010;9:161-7.

34 Phung V-H, Trueman I, Togher F, et al. Perceptions and experiences of community first responders on their role and relationships: qualitative interview study. Scand J Trauma Resusc Emerg Med 2018;26:13

35 Barry T, Guerin S, Bury G. Motivation, challenges and realities of volunteer community cardiac arrest response: a qualitative study of 'lay' community first responders. BMJ Open 2019;9:e029015.

36 Barry T, Guerin S, Headon M, et al. GPs who volunteer to be first responders for out-of-hospital cardiac arrest: a qualitative study. Eur $J$ Gen Pract 2020;26:33-41.

37 Ives J, Damery S. Qualitative data collection. In: Walker DM, ed. An introduction to health services research. London: Sage, 2014.

38 Malterud K. Qualitative research: Standards, challenges, and guidelines. Lancet 2001;358:483-8.

39 O'Brien BC, Harris IB, Beckman TJ, et al. Standards for reporting qualitative research: a synthesis of recommendations. Acad Med 2014;89:1245-51.

40 Johnson RB, Onwuegbuzie AJ. Mixed methods research: a research paradigm whose time has come. Educ Res 2004;33:14-26.

41 Starks H, Trinidad SB, Brown Trinidad S. Choose your method: a comparison of phenomenology, discourse analysis, and grounded theory. Qual Health Res 2007:17:1372-80.

42 Brinkmann S. Unstructured and semi-structured interviewing. In: Leavy P, ed. The Oxford handbook of qualitative research. Oxford: Oxford Library of Psychology, 2014: 277-99.

43 Britten N. Qualitative research: qualitative interviews in medical research. BMJ 1995;311:251-3.

44 Yardley L. Demonstrating validity in qualitative psychology. In: Smith JA, ed. Qualitative psychology: a practical guide to research methods. London: Sage Publications, 2008: 235-51.

45 Carter N, Bryant-Lukosius D, DiCenso A, et al. The use of triangulation in qualitative research. Oncol Nurs Forum 2014:41:545-7.

46 Patton MQ. Qualitative evaluation and research methods. Beverly Hills, CA: Sage, 1990: 169-86.

47 Kuper A, Lingard L, Levinson W. Critically appraising qualitative research. BMJ 2008;337:a1035.

48 Heffernan E, Coulson NS, Henshaw $\mathrm{H}$, et al. Understanding the psychosocial experiences of adults with mild-moderate hearing Ioss: an application of Leventhal's self-regulatory model. Int J Audiol 2016;55:S3-12.

49 Pearson J, Hammond M, Heffernan E, et al. Careers and talents not to be wasted. J Manag Dev 2012;31:102-15.

50 Heffernan E, Coulson NS, Ferguson MA. Development of the social participation restrictions questionnaire (SPaRQ) through consultation with adults with hearing loss, researchers, and clinicians: a content evaluation study. Int J Audiol 2018;57:791-9.

51 Braun V, Clarke V. Using thematic analysis in psychology. Qual Res Psychol 2006;3:77-101.

52 Graneheim UH, Lindgren B-M, Lundman B. Methodological challenges in qualitative content analysis: a discussion paper. Nurse Educ Today 2017;56:29-34.

53 Creswell JW, Miller DL. Determining validity in qualitative inquiry. Theory Pract 2000;39:124-30.

54 Morse JM. Critical analysis of strategies for determining rigor in qualitative inquiry. Qual Health Res 2015;25:1212-22.

55 Timmons S, Vernon-Evans A. Why do people volunteer for community first responder groups? Emerg Med J 2013;30:e13.

56 Roberts A, Nimegeer A, Farmer J, et al. The experience of community first responders in co-producing rural health care: in the liminal gap between citizen and professional. BMC Health Serv Res 2014:14:460.

57 Mathiesen WT, Bjørshol CA, Braut GS, et al. Reactions and coping strategies in lay rescuers who have provided CPR to out-ofhospital cardiac arrest victims: a qualitative study. BMJ Open 2016;6:e010671.

58 Møller TP, Hansen CM, Fjordholt M, et al. Debriefing bystanders of out-of-hospital cardiac arrest is valuable. Resuscitation 2014;85:1504-11.

59 O'Meara P, Tourle V, Rae J. Factors influencing the successfu integration of ambulance volunteers and first responders into ambulance services. Health Soc Care Community 2012;20:488-96.

60 Kindness P, Fitzpatrick D, Mellish C, et al. An insight into the demands and stressors experienced by community first responders. J Paramed Pract 2014;6:362-9. 
61 Zijlstra JA, Beesems SG, De Haan RJ, et al. Psychological impact on dispatched local lay rescuers performing bystander cardiopulmonary resuscitation. Resuscitation 2015;92:115-21.

62 Peberdy MA, Ottingham LV, Groh WJ, et al. Adverse events associated with lay emergency response programs: the public access defibrillation trial experience. Resuscitation 2006;70:59-65.

63 Snobelen PJ, Pellegrino JL, Nevils GS. Helping those who help: the lay Responder post-arrest support model. Circ Cardiovasc Qual Outcomes 2018;11:e004702.

64 Bailey S, Savage S, OConnell B. Volunteering and social capital in regional Victoria. Aust J Volunt. 2003;8:5-12.

65 Poortinga W. Social relations or social capital? Individual and community health effects of bonding social capital. Soc Sci Med 2006;63:255-70.
66 Mohnen SM, Groenewegen PP, Völker B, et al. Neighborhood social capital and individual health. Soc Sci Med 2011;72:660-7.

67 Bremer A, Dahlberg K, Sandman L. Balancing between closeness and distance: emergency medical services personnel's experiences of caring for families at out-of-hospital cardiac arrest and sudden death. Prehosp Disaster Med 2012;27:42-52.

68 United Nations Development Programme. Human development report 2019 - Beyond income, beyond averages, beyond today: Inequalities in human development in the 21st century. New York, 2019.

69 Svensson A, Fridlund B, Wångmar E, et al. Home healthcare nurses' experiences of being on stand by as a first responder in a 'while waiting for the ambulance' assignment. Nord J Nurs Res 2016;36:184-91. 